\title{
The role of detection and recollection of change in list discrimination
}

\author{
Larry L. Jacoby • Christopher N. Wahlheim • \\ Andrew P. Yonelinas
}

Published online: 26 March 2013

(C) Psychonomic Society, Inc. 2013

\begin{abstract}
In two experiments, we examined the importance of the detection and recollection of change for list discrimination. Two lists of pairs were presented, with the right-hand member being changed between lists for some pairs. Participants in Experiment 1 were instructed to explicitly indicate when they detected a change between pairs during the presentation of List 2, whereas participants in Experiment 2 were not instructed to do so. At the time of test, participants in both experiments were presented with a pair and asked whether it had been presented in List 2. Next, recollection of change was measured by asking whether the right-hand member of the pair was changed between the lists. The results from Experiment 1 revealed high correspondence between the detection of change during the presentation of List 2 and the recollection of change at the time of test. Consequently, change recollection at test can serve as a measure of earlier change detection, in combination with access to memory for change at the time of test. In both experiments, as compared to control conditions, proactive facilitation in list discrimination was observed when change was recollected, whereas proactive interference was observed when change was not recollected. These results were interpreted as showing that recursive reminding - bringing a List 1 pair to mind during the presentation of its changed List 2 pair-embeds memory for the earlier event into memory for the later event, and doing so preserves information about list membership.
\end{abstract}

L. L. Jacoby $(\bowtie) \cdot$ C. N. Wahlheim

Department of Psychology, Washington University in St. Louis, One Brookings Drive,

St. Louis, MO 63130, USA

e-mail: 1ljacoby@artsci.wustl.edu

A. P. Yonelinas

Department of Psychology, University of California,

Davis, CA, USA
Keywords Change detection - Proactive effects · List discrimination $\cdot$ Recursive remindings $\cdot$ Source memory

Suppose that you witnessed two political debates, and a candidate who made a statement about an issue in the first debate contradicted that statement in the second debate. Later, you are presented with either the first or the second statement and asked whether the statement originated from the second debate. Would your task be made easier if you detected the contradiction between the candidate's statements while watching the second debate and recollected that change at the time of test? The detection and recollection of change concern memory for the relationships among events (e.g., what is the relationship between what was said in the first vs. the second debate). We argue that recursive reminding plays an important role in memory for change. In the example of the self-contradicting politician, recursive reminding would refer to the first position held by the politician coming to mind during his or her advocating a second position, with the result that the change is detected. For detection of change to later be important, change must be recollected at the time of test. To anticipate, results from the experiments that are to be reported here revealed the importance of detection and recollection of change for list discrimination.

In the language of paired-associate learning, the case of the self-contradicting politician corresponds to an A-B, A-D paradigm (i.e., the same politician paired with different responses on the two occasions). The question regarding whether or not a position was stated in the second debate corresponds to a question about list discrimination, which is akin to questioning the recency of the debate in which a statement occurred. The results from investigations of within-list recency judgments have been interpreted as being due to recursive reminding: Judgments of presentation recency are superior for related (e.g., queen-king) as compared to 
unrelated (e.g., spider-table) words (Hintzman, 2010; Tzeng \& Cotton, 1980; Winograd \& Soloway, 1985). Hintzman (e.g., 2010) has argued that reminding results in a recursive representation in which the first-presented member of a related pair is embedded in the memory of the second-presented member, preserving their temporal order. For example, remembering that "king" reminded one of "queen" allows one to be certain that "king" was presented more recently than was "queen."

Jacoby and Wahlheim (2013) provided evidence that detection of the shared category membership of words can be brought under task control by means of a looking-back procedure in which, for one condition, the detection of the relation between items from a pair (e.g., table-couch) that shared category membership was encouraged, and in another condition, detection of the relation between items from a pair was discouraged. The accuracy of recency judgments for presentation of pair members was shown to be increased when the detection of shared category membership was encouraged as compared to discouraged. Additional results revealed that effects on recall of the most recently presented member of a category when given the category label as a cue (e.g., "an article of furniture" as a cue for recall of "couch") paralleled effects on recency judgments. For cued-recall performance, recursive reminding allowed participants to avoid errors, as was shown by the correspondence between errors in recency judgments and intrusion errors in cued recall. These results provided evidence that both recency judgments and cued-recall performance relied on the recollection of recursive reminding.

Wahlheim and Jacoby (2013) introduced their article with an example of recalling contradictory statements made by a politician that was similar to the one used to begin the present article. In their experiments, participants studied two lists of word pairs that included pairs with the same cue and a changed response (A-B, A-D), as well as control pairs presented exclusively in the second list $(\mathrm{A}-\mathrm{B}, \mathrm{C}-\mathrm{D})$. At test, participants were provided with the left-hand member of pairs as cues for recall of the right-hand member presented in the second list. As will be described later, recollection of change was measured by means of a remindings-report procedure. The results showed that performance on A-D pairs depended on whether or not change was recollected: Proactive facilitation was observed when change was recollected, whereas proactive interference was observed when change was not recollected.

The goal of the present experiments was to show that list discrimination performance parallels cued-recall performance in showing effects of change recollection, as was found for recency judgments and cued-recall performance (Jacoby \& Wahlheim, 2013). An account of proactive effects on list discrimination in terms of recollection of change contrasts with earlier accounts that have emphasized the importance of list differentiation. Proactive interference has been described as resulting from competition between responses paired with the same cue word at the time of test (e.g., Postman \& Underwood, 1973). Winograd (1968) examined participants' ability to identify the list membership of previously studied items as a means of resolving response competition. The results of his experiments revealed the importance of relative frequency by showing that participants' ability to identify the list from which a tested item originated was poorest when the two lists were presented equally often. This was said to reduce the ability to use differences in memory strength as a basis for list discrimination. A second means of accomplishing list discrimination has been said to rely on associations between words and their list context. Such associations have been described as being an abstract list tag or label that is associated with but not intrinsic to the content of a memory (Anderson \& Bower, 1974; Murnane, Phelps, \& Malmberg, 1999).

Results reported by Wahlheim and Jacoby (2013) suggested that recollection of change serves as a third basis for list discrimination. Recursive reminding provides a basis for later list discrimination by embedding memory for the List 1 response in memory of the List 2 response, and thereby preserving the order in which they occurred. Returning to the example of a self-contradicting politician, if one is reminded of a statement made in a first debate by a contradictory statement made in the second debate, recollection of that reminding preserves the order of the two statements. One can be certain that the contradictory statement occurred during the second debate; otherwise, it could not have brought the earlier statement to mind. Similarly, detection and recollection of a between-list change in the response paired with a cue serves as a basis for list discrimination.

In the present experiments, we investigated the effects of the detection and recollection of change on subsequent list discrimination by employing a within-subjects manipulation of the correspondence between List 1 and List 2 pairs. The conditions that were employed are illustrated in Table 1. For example, "changed-target" refers to a condition for which responses were changed across lists, with the List 2 pair

Table 1 Distribution of pairs across phases for each item type

\begin{tabular}{llll}
\hline & \multicolumn{2}{l}{ Phase } & \\
\cline { 2 - 3 } Item Type & List 1 & List 2 & Test \\
\hline Changed-target & card-paper & card-poker & card-poker (target) \\
Changed-foil & knee-bone & knee-bend & knee-bone (foil) \\
Control-target & - & cherry-pie & cherry-pie (target) \\
Control-foil & limp-walk & - & limp-walk (foil) \\
Repetition & eagle-bald & eagle-bald & eagle-bald (target) \\
New foil & - & - & lamb-wool (foil) \\
\hline
\end{tabular}


being presented at test for participants to judge whether or not it had been presented in List 2. A "yes" response in that condition would count as a "hit." In contrast, for the "changed-foil" condition, the List 1 pair was presented at test, and a "yes" response to the question about its having occurred in List 2 would count as a "false alarm." List discrimination was measured in terms of hits and false alarms in the changed conditions (i.e., the conditions in which reminding could occur), and this was compared to the difference between hits and false alarms in the control conditions (i.e., the conditions in which reminding could not occur). Additional conditions were a "repetition" condition, in which pairs were repeated across the two lists, and a "new foil" condition, in which pairs had not been presented in either of the two lists but were presented at test.

At test, recollection of change was measured by presenting a test pair and asking participants whether the right-hand member of the pair had changed between Lists 1 and 2. This is a more direct measure of recollection of change than the remindings-report procedure used in earlier experiments (Jacoby \& Wahlheim, 2013; Wahlheim \& Jacoby, 2013). For the remindings-report procedure, participants were instructed that if another word came to mind prior to or simultaneously with a word that they produced as being a List 2 response at test, they were to report the word that came to mind. Reporting a List 1 response as having come to mind was treated as indicating that a reminding that had occurred during List 2 study was recollected at test.

Experiment 1 was aimed at showing that the new recollection-of-change measure (asking participants at the time of test whether the right member of a pair had been changed earlier) reflected change detection during the presentation of List 2. Participants in Experiment 1 were instructed that during the presentation of List 2, they were to indicate if they noticed that the right-hand member of a presented pair was changed between the lists (i.e., A-B, $\mathrm{A}-\mathrm{D}$ items). Their doing so for changed pairs was treated as a measure of change detection. Furthermore, they were told to recall the List 1 response when they detected such a change. Correct recall of the List 1 response would provide further evidence that change had been detected. A finding that recollection of change, measured at test, relied on detection of change during the presentation of List 2 would provide evidence that the recollection-of-change measure reflects earlier detection of change.

Using the remindings-report procedure, Wahlheim and Jacoby (2013) found that the probability of recollecting change at the time of test was much lower than was the probability of detecting change during the presentation of List 2. That result was interpreted as showing that the recursive trace produced by the detection of change was sometimes lost or not accessed at the time of test. However, a difficulty for that interpretation is that the measure of detection of change during the presentation of List 2 differed greatly from the measure of recollection of change at test. In contrast, the two measures were very similar in the present experiments, allowing loss of access to memory for change to be measured more accurately.

Wahlheim and Jacoby (2013) found that cued-recall performance for changed pairs depended on the recollection of change. We expected to find similar results for list discrimination performance. When change was not recollected, we expected proactive interference in list discrimination performance for the changed conditions as compared to the control conditions. In contrast, when change was detected and recollected, we expected proactive facilitation in the form of superior list discrimination performance. This is because we expected recollection of change to both increase the probability of a hits and decrease the probability of a false alarms in list discrimination performance: Access to memory for a recursive reminding that embeds the List 1 pair $(\mathrm{A}-\mathrm{B})$ in memory for the List 2 pair (A-D) and preserves their order of occurrence provides both a basis for hits and a sure basis for rejecting the List 1 pair as having occurred in List 2.

The predicted pattern of results could not be easily accounted for by appealing either to differences in familiarity (e.g., Winograd, 1968) or to list tags (e.g., Anderson \& Bower, 1974). Neither of those two bases for list discrimination would preserve memory for change. High familiarity of a tested pair provides a basis for accepting an item as having occurred in List 2, but it does not serve as a sure basis for rejection of pairs that occurred in List 1. Similarly, the attachment of a list tag that identifies a tested pair as being presented in List 2 gives reason to accept it as having occurred in List 2, but the presence of a List 1 tag would not give a sure basis for rejecting a List 1 pair that originated from the changed condition, because of the presence of pairs that occurred in both lists (repetition pairs). Also, list tags do not preserve information about change, although a comparison of list tags could be used to detect change at the time of test. In contrast, access to a recursive representation of change would make it unnecessary to compute change at the time of test. We find it untenable to argue that people cannot detect change when it occurs and later recollect the change, but instead must later compare list tags to discover that change had earlier occurred. Again, although familiarity and list tags can serve as bases for hits, as can recollection of change, only recollection of change would provide a sure means of avoiding false alarms.

As described above, a goal of Experiment 1 was to show that the recollection-of-change measure used at test reflected detection of change during the presentation of List 2. Doing so would require that participants explicitly detect change during the presentation of List 2. However, a potential difficulty is that explicitly detecting change during presentation of List 2 might produce a relationship between change 
detection and change recollection that would not otherwise be observed. For example, change detection might occur only if participants are explicitly instructed to detect change. Against this possibility, we expected the effects of recollection of change on list discrimination performance to be the same in Experiments 1 and 2, the latter of which did not require explicit detection of change during the presentation of List 2. Finding that effects were the same in the two experiments would show that participants detected change, even when they were not told to do so.

Conclusions from our experiments would rely on the use of conditional probabilities to analyze the effects of recollection of change on list discrimination performance. Doing so would produce a risk that item selection effects would contribute to our results. Wahlheim and Jacoby (2013) employed hierarchical multiple regression analyses to show that recollection contributes to cued-recall performance beyond item differences, and to show large individual differences in the contribution of recollection of change to cuedrecall performance. We employed similar analyses and expected to find corresponding effects for list discrimination performance.

\section{Experiment 1}

\section{Method}

Participants Thirty-six Washington University students participated in exchange for course credit or $\$ 10 / \mathrm{h}$. All participants were tested individually.

Design and materials A 6 (item type: changed-target, changed-foil, control-target, control-foil, repetition, and new foils) within-subjects design was used. The critical materials consisted of 120 three-word sets that included a cue word (e.g., knee) and two responses associated with the cue (e.g., bone, bend). These sets were drawn from Jacoby (1996) and Nelson, McEvoy, and Schreiber (1998). The responses in each set had several overlapping letters, because they were originally designed to create fragments that could be completed by either of the two responses (e.g., $b$ n_ could be completed by bone or bend). The forward and backward associative strengths between the cues and responses were low on average (forward, $M=.05, S D=$ .09 ; backward, $M=.08, S D=.15$ ), and the same was true for associations between the responses (forward, $M=.02$, $S D=.05$; backward, $M=.03, S D=.08$ ), as indexed by Nelson et al.

Table 1 displays the distribution of pairs across each phase of the experiment for each of the six pair types. Six groups of 20 sets served as the critical items. The assignment of critical items to conditions was counterbalanced such that, across participants, they equally often represented the within-subjects conditions. An additional six groups of two sets (12 pairs) remained constant across formats and were used as primacy and recency buffers and as practice items at test. List 1 contained 88 pairs that consisted of 80 critical items (20 changed-target, 20 changed-foil, 20 control-foil, and 20 repetition) and eight buffers (two changed-target, two changed-foil, two control-foil, and two repetition) split evenly between the primacy and recency positions in the list. List 2 contained 88 pairs that consisted of 80 critical items ( 20 changed-target, 20 changed-foil, 20 control-target, and 20 repetition) and eight buffers (two changed-target, two changed-foil, two control-target, and two repetition), again split evenly between the primacy and recency positions in the list. List 2 contained twice as many pairs representing the changed conditions (40), as compared to the control-target and repetition conditions (20 each). This was necessary to produce changed-target and changed-foil conditions with 20 pairs representing each of the conditions. At test, the 12 pairs reserved for buffers appeared on a practice test, two from each condition. Following that, the actual test contained all 120 critical items. Note that our counterbalancing scheme resulted in items appearing equally often as control and changed pairs, which allowed us to perform the regression analyses described in the introduction.

Procedure List 1 pairs appeared individually in a fixed random order, with the restriction that items from the same condition did not appear consecutively more than three times. Pairs appeared once each for $3 \mathrm{~s}$, followed by a 500-ms interstimulus interval (ISI). Participants were told to study the pairs for an upcoming memory test.

List 2 pairs appeared with the same restrictions as for List 1. Participants' first task was to study for as long as was necessary to learn each pair completely for an upcoming test. Their second task was to indicate pairs for which the responses had changed (changed pairs) and to recall the right-hand member of the List 1 pair that corresponded to the changed pair. Boxes labeled "next" and "right word changed" appeared below the pairs. Participants were told to click "next" when they had completed studying an unchanged pair, or to click "right word changed" when they noticed that the presented List 2 pair was changed from a List 1 pair. After indicating change, the participants attempted to recall the List 1 response by typing their response onto the screen and then pressing the "Enter" key. If unable to recall the List 1 response, participants were instructed only to press the "Enter" key. After participants pressed "Enter," the List 2 pair remained on the screen with only the "next" box. Participants were instructed to continue studying the List 2 pair until it was learned completely, at which point they clicked "next" to move on. 
At test, pairs again appeared in a fixed random order, with the same restrictions as in the previous lists. Participants' first task was to indicate whether the pair had been presented in List 2 by clicking on boxes labeled "yes" or "no." A "no" response was to be made if the pair either had appeared in List 1 or was new to the experiment. Their second task was to indicate whether the right-hand member had changed from List 1 to List 2 while the left-hand member remained the same, by clicking on boxes labeled in the same manner as for the previous judgment. Pairs remained on the screen until participants had made a response.

\section{Results and discussion}

Significant effects were those below $\alpha=.05$. However, we will also report nonsignificant effects that are of theoretical importance. Variations in the degrees of freedom and means for pairwise comparisons in conditional analyses are the result of excluding participants who did not have at least one observation in each cell.

Hits and false alarms were indexed as "yes" responses at test for pairs that had (hits) and had not (false alarms) been presented in List 2 . The probability of a hit in the repetition condition was very high, whereas the probability of a false alarm to new foils was quite low (.93 vs. .05). Of primary interest were the differences in list discrimination indexed by hits and false alarms in the changed and control conditions. The left panel of Fig. 1 shows that the probability of a hit was higher in the changed-target than in the controltarget condition, $t(35)=4.02, p<.001$, whereas no significant difference in false alarms appeared between the changed-foil and control-foil conditions, $t(35)=-0.53, p=.60$. A list discrimination measure, $d^{\prime}$ (Macmillan \& Creelman, 2005), showed better discrimination for the changed than for the control conditions (2.07 vs. 1.70$), t(35)=2.53, p=.02$, indicating proactive facilitation for list discrimination of changed items. As we argued in the introduction, we expected list discrimination performance in the changed conditions to reflect a mixture of proactive facilitation and proactive interference, dependent on whether the change had been recollected at the time of test. Later, we will describe evidence to show that the results in Fig. 1 do reflect a mix of that sort.

Use of the self-paced procedure during the presentation of List 2 resulted in differences in study times. We do not report those differences, because they are difficult to interpret. For example, it is not possible to truly separate the contribution of the time taken to detect change from that of the time spent attempting to recall the List 1 response for changed items. As will be seen, in Experiment 2 we used a fixed rate of study time and replicated the results of Experiment 1.

Relationship between change detection and change recollection Participants rarely erroneously indicated detection of change for the repetition and control pairs (.03 and .04 , respectively) during the presentation of List 2 . The overall probability of change detection during List 2 presentation in the changed conditions was not high (.57). However, List 1 responses were recalled with a high probability when change was detected (.87), providing further evidence that List 1 responses did come to mind during the presentation of changed pairs in List 2 .

As is shown in Table 2, the probabilities of reporting change at test when change had not been detected in List 2 were quite low and did not differ significantly between the changed-target and control-target conditions, $t(35)=0.77$, $p=.45$. When change was detected during List 2 , there was a very high probability of the change being recollected at the time of test (.93), showing that the measure of change
Fig. 1 Hit and false alarm rates in list discrimination judgments for changed and control pairs in Experiment 1 (left panel) and Experiment 2 (right panel). Bars represent the standard errors of the means

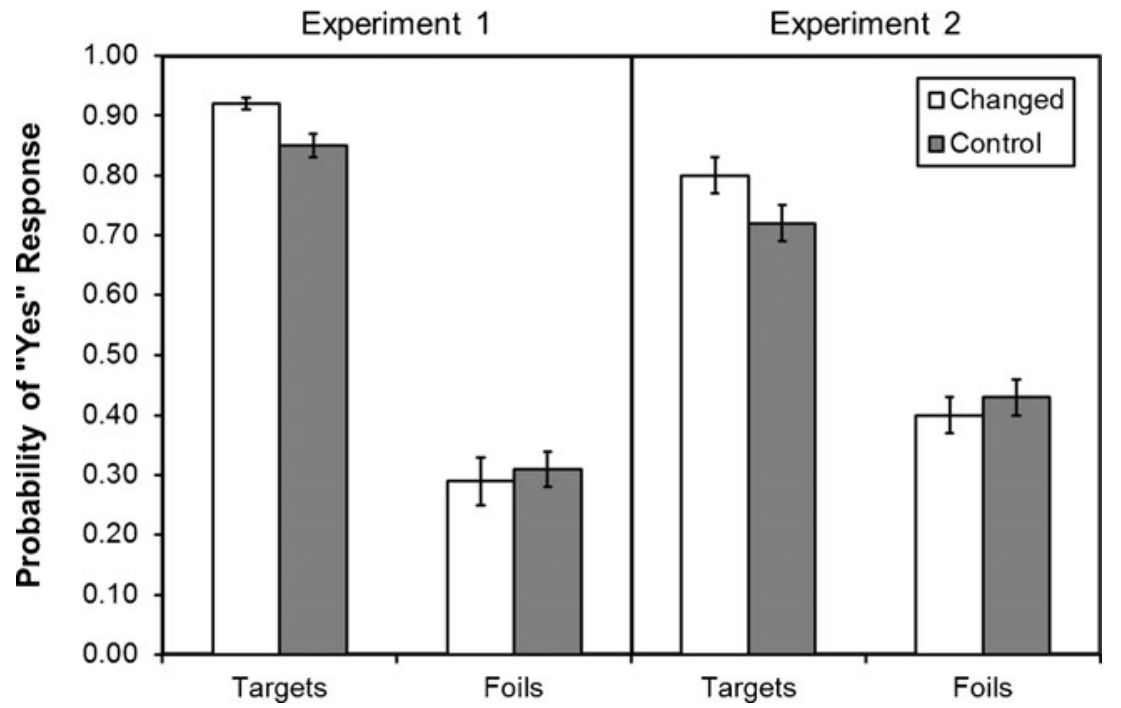


Table 2 Experiment 1: Probability of reporting a changed pair at test, conditionalized on change detection in List 2

\begin{tabular}{lccc}
\hline & \multicolumn{3}{l}{ Change Detection (List 2) } \\
\cline { 2 - 4 } Item Type & Changed (Yes) & Changed (No) & Control (No) \\
\hline Target & $.93(.01)$ & $.12(.03)$ & $.10(.02)$ \\
Foil & $.93(.02)$ & $.43(.04)$ & $.06(.01)$ \\
\hline
\end{tabular}

Standard errors of the means are displayed in parentheses

recollection accurately reflected change detection. When change was not detected during List 2 , the probability of saying that a pair had changed at test was higher in the changed-foil than in the changed-target condition, $t(34)=$ $6.57, p<.001$. This difference likely arose because the participants in the changed-foil condition were more likely to first detect change at the time of test, because of the close proximity of changed foils at test to corresponding pairs presented in the immediately preceding List 2 . For the changed-target condition, changed pairs presented at test were farther distant from the List 1 presentation of the corresponding pairs. It is likely that the demand to explicitly detect change during the presentation of List 2 contributed to the probability of first detecting change at the time of test.

In contrast to the results reported by Wahlheim and Jacoby (2013), the probability of change recollection differed little from the probability of change detection, showing little forgetting or failure to access memory across the delay between change detection and the test of change recollection. That difference in results might reflect the different methods used to measure recollection of change. However, in part, the difference is likely due to the test of list discrimination providing more cues for the recollection of change (a complete pair) than did the test of cued recall employed by Wahlheim and Jacoby (only the left-hand member of a pair). It seems likely that access to memory for change depends on the cues provided at test and on the delay between detection of change and the test of its recollection.

Change recollection and list discrimination Collapsed across probabilities of detecting change in List 2, the probability of participants reporting that responses had changed at the time of test was higher in the changed-foil than in the changed-target condition (.72 vs. .57), $t(35)=4.82$, $p<.001$. As we indicated earlier, that difference is likely due to the higher probability in the changed-foil condition of first detecting change at the time of test. The probability of incorrectly reporting that pairs had changed was lower in the control-foil than in the control-target condition (.06 vs. .10), $t(35)=-2.37, p=.02$.

Conditionalizing list discrimination performance on recollection of change (Fig. 2) provides evidence of a mixture of proactive facilitation and proactive interference effects. The probability of a hit in list discrimination for changedtarget test pairs was higher when participants recollected change at test than when they did not (.96 vs. .87), and was also higher than the probability of a hit for controltarget test pairs (.96 vs. .85$), t \mathrm{~s}(35) \geq 4.27, p \mathrm{~s}<.001$. When change was not recollected, the probabilities of a hit did not differ significantly between the changed-target and controltarget pairs (.87 vs. .85), $t(35)=-1.04, p=.31$. Although differences in the probabilities of a hit were small, large differences were evident in the probability of false alarms in list identification performance. The probability of a false alarm to changed-foil test pairs was much lower when change was recollected than when it was not. When change was not recollected, fewer false alarms occurred for control foils than for changed foils (.31 vs. .56), $t(33)=-7.64$, $p<.001$. In contrast, when change was recollected, the opposite was found (.19 vs. .31), $t(35)=-2.23, p=.03$.

Most importantly, list discrimination performance, measured by $d^{\prime}$, provides evidence of proactive facilitation when change was recollected and proactive interference when change was not recollected. List discrimination was higher in the changed conditions when change was recollected than in the control conditions (2.54 vs. 1.70$), t(35)=4.35, p<.001$, but was lower than in the control conditions when change was not recollected ( 0.92 vs. 1.68$), t(33)=-6.66, p<.001$.

Note that the major differences in the results shown in Fig. 2 are differences in false alarms rather than in hits. This likely occurred because hits were sometimes a result of relying on differences in familiarity or, perhaps, list tags, as well as recollection of change as a basis for responding that a tested pair had occurred in List 2, limiting the opportunity for recollection of change to have an effect. In contrast, recollection of change served as a sure basis for correctly rejecting a List 1 pair as having occurred in List 2, whereas responding on the basis of familiarity or list tags did not do so. Again, recollecting that one was reminded of a List 1 pair during the presentation of a List 2 pair allows one to be certain that the List 1 pair did not appear in List 2. The finding that the probability of false alarms in list identification performance was much higher in the changed-foil condition when change was not recollected than in the control-foil condition likely reflects the greater familiarity of the left-hand member of a test pair, produced by its repetition across lists. In the changed-foil condition, the greater familiarity of a test pair was successfully opposed when change was recollected, but instead mistaken as having originated from presentation of the pair in List 2 when change was not recollected.

Regression analyses Results from the analysis above relied on conditionalized data, and so raise the concern that item differences might have played a role in producing the 
Fig. 2 Hit and false alarm rates in list discrimination judgments for changed pairs, conditionalized on change recollection, and for control pairs in Experiment 1 (left panel) and Experiment 2 (right panel). Bars represent the standard errors of the means

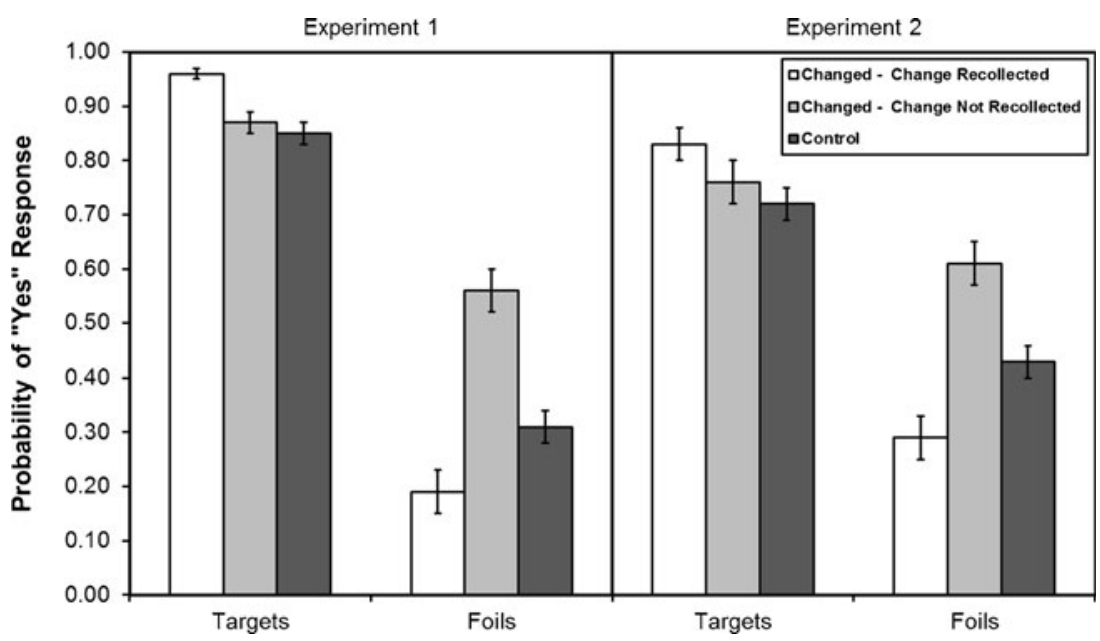

results. For example, some items may be more salient, with the result that their list membership is remembered better, regardless of whether or not change is recollected. We employed a hierarchical multiple regression analysis to examine effects on list discrimination performance for changed pairs, beyond any effects produced by item differences. On the basis of findings from earlier experiments, we expected that both item differences and change recollection would predict list discrimination performance $\left(d^{\prime}\right)$ for changed pairs, but that change recollection would explain unique variance when controlling for item differences (Jacoby \& Wahlheim, 2013; Wahlheim \& Jacoby, 2013).

Table 3 displays the regression results. In the model conducted at the item level, we entered list discrimination performance for control pairs (as $d^{\prime}$ ) at the first step, to measure the contribution of differences in memory for items to list discrimination performance on changed pairs. Doing so is justified because, across formats, pairs were rotated through conditions, and so, pairs were the same for the control and changed conditions. At the second step, we entered a composite score for change recollection. The probability of correctly judging that responses had changed across lists was computed as the probability of a hit for changed pairs minus the probability of incorrectly identifying items as having changed (false alarms) for control pairs. ${ }^{1}$

The top panel of Table 3 shows that item differences accounted for a small but significant proportion of variance in list discrimination at the first step. More importantly, when controlling for item differences, change recollection

\footnotetext{
$\overline{{ }^{1} \text { We used hits }}$ - false alarms to produce a corrected measure of change recollection, whereas we used a $d^{\prime}$ measure of list discrimination performance. We did so because we assumed that recollection of change was all-or-none, whereas list discrimination had a continuous basis because of the contribution of familiarity (cf. Yonelinas, 2001). However, the choice among change measures is not crucial. Use of a $d^{\prime}$ measure of change recollection produced results that were in complete agreement with those obtained using the hits - false alarms measure.
}

significantly improved prediction at the second step. The interaction term did not improve prediction. These results show that item differences in memory for individual pairs contributed little to list discrimination performance. However, some items supported change recollection more than others did, and this difference in change recollection contributed greatly to list discrimination performance. Change recollection explained variance in list discrimination performance above and beyond the effects due to item differences.

Wahlheim and Jacoby (2013), as well as Jacoby and Wahlheim (2013), found individual differences among

Table 3 Experiments 1 and 2: Proportions of explained variance in list discrimination for changed pairs

Experiment

12

Item Level
Step 1: Item differences

Step 2: Change recollection

Step 3: Interaction

Participant Level

Step 1: General discrimination

Step 2: Change recollection

Step 3: Interaction

$\begin{array}{ll}.05^{*} & .00 \\ .15^{* *} & .22^{* *} \\ .01 & .01\end{array}$

$.19^{* *}$

$.27^{* *}$

.00
The values displayed above are $\Delta R^{2}$ values at each step of the model, computed at the item level collapsed across participants (top), and at the participant level collapsed across items (bottom). "Item differences" refers to individual differences in the $d^{\prime}$ values for control items at the item level, and "General discrimination" refers to the same probabilities computed at the participant level. "Change recollection" refers to the probability of correctly saying "yes" that changed pairs from Lists 1 and 2 had indeed changed minus the probability of incorrectly saying "yes" that the corresponding control items had changed, at the item and participant levels. "Interaction" refers to the interaction term for the aforementioned predictor variables. ${ }^{*} p<.05$. ${ }^{* *} p<.01$ 
participants in their probabilities of change recollection. We examined the contribution of individual differences in change recollection by using a regression model similar to that used to examine the influence of item differences. The primary difference between models was that instead of items, participants were the unit of analysis. Predictors were entered in the same manner as in the previous model, except that the first step included individual differences in general discrimination ability, as measured by list discrimination performance on the control pairs. The results revealed that general discrimination ability explained a significant proportion of variance in list discrimination. Individual differences in change recollection, entered at the second step, significantly improved the prediction of list discrimination performance after controlling for individual differences in general discrimination ability. The interaction term did not improve prediction. These results provide evidence of large individual differences in the use of change recollection as a basis for list discrimination.

\section{Experiment 2}

A potential difficulty in interpreting the results from Experiment 1 is that requiring explicit detection of change during the presentation of List 2 might have influenced the list discrimination results by means other than an influence on recollection of change. To examine that possibility, explicit change detection during the presentation of List 2 was not required in Experiment 2. Not doing so made it possible to present List 2 pairs at a fixed rate in Experiment 2, allowing us to show that the pattern of list discrimination results observed in Experiment 1 did not result from differences in study time produced by allowing self-allocation of study time. Despite these changes in procedure, we expected the results of Experiment 2 to replicate those of Experiment 1, in that list discrimination performance in the changed conditions should show proactive facilitation when change was recollected and proactive interference when change was not recollected. However, we did anticipate that removing the requirement of detecting change explicitly during the presentation of List 2 would reduce the probability of change detection, and so, reduce change recollection at the time of test.

An additional difference between the experiments was that a manipulation meant to influence the detection of change during the presentation of List 2 was included in Experiment 2. Detection of change during List 2 was made more difficult in this experiment than in Experiment 1 by inserting a 2-min delay between the presentation of the lists, during which time participants were instructed to write down things that they would do if they were invisible and not responsible for their actions. This intervening task was shown by Sahakyan and Kelley (2002) to reduce proactive interference. Participants in a "reminding" condition were instructed to think back across the intervening task to List 1 during the presentation of List 2, so as to detect pairs in which the right-hand member had been changed (changed items). They were told that following the presentation of List 2, they would be asked to estimate the proportion of presented pairs that had been changed between lists. The procedure for a "differentiation" condition was the same as for the reminding condition, but the instructions discouraged participants from thinking back to List 1 during the presentation of List 2. For the differentiation condition, participants were instructed that they would receive a second list of pairs that they would be asked to remember and that fully engaging in the invisibility task would help them forget the earlier list, and thereby improve their memory performance for the later list. They were not informed that List 2 would include changed pairs, and so were not told that they would later be asked to estimate the proportion of pairs for which responses had been changed between lists. We expected the manipulation of instructions to produce higher recollection of change in the looking-back than in the differentiation condition, which would show that, in part, detection of change can be brought under task control (cf. Jacoby \& Wahlheim, 2013).

\section{Method}

Participants Thirty-six Washington University students participated in exchange for course credit or $\$ 10 / \mathrm{h}$. Eighteen of the participants were randomly assigned to each of the two conditions that were produced by a manipulation of instructions meant to influence the probability of change detection during List 2. All participants were tested individually.

Design, materials, and procedure The design, materials, and procedure were identical to those of Experiment 1, with the noted exceptions. Prior to List 1, participants in both instruction conditions were told to read the word pairs aloud and to study them for an upcoming memory test. In contrast to Experiment 1, a 2-min intervening task was introduced between Lists 1 and 2. After the presentation of List 1, participants were instructed to write down what they would do if they were invisible and were not responsible for their actions (cf. Sahakyan \& Kelley, 2002). A differentiation group were told that they would be presented with another list of words that they would need to remember for an upcoming test, and that fully engaging themselves in the invisibility task would help them forget the earlier list, and thereby improve their memory for the later list. They were not informed about the nature of the relationships between pairs in Lists 1 and 2. In contrast, a remindings group were told that they should think back to List 1 during the presentation of List 2, so as to note changed pairs. Furthermore, 
they were told that following the presentation of List 2, they would be asked to estimate the proportion of pairs in List 2 that had been changed. Participants in the differentiation group were not warned about that upcoming test. The List 2 study duration was fixed at $5 \mathrm{~s}$ per pair for both groups. Following List 2 study, both groups first made aggregate estimates of the percentage of pairs that had changed between lists, and then they made the same estimates for the pairs that had repeated across lists.

At test, pairs were presented in the same manner as in Experiment 1. However, participants in Experiment 2 made their judgments regarding List 2 membership by using a 6point scale. Below each word pair appeared the prompt "List 2 ?" and six buttons. The lower-bound value on the scale, 1 , indicated certainty that a pair had not appeared in List 2 (sure "no"). In contrast, the upper-bound value 6 indicated certainty that a pair had appeared in List 2 (sure "yes"). The intermediate values represented varying degrees of certainty, with the least certain responses falling on the values of 3 and 4 . Participants clicked on the button that represented their level of certainty and then made judgments of change in the same manner as in Experiment 1.

\section{Results and discussion}

To facilitate comparisons of the results across experiments, responses of 4-6 on the 6-point scale used for judgments of list membership were treated as "yes" responses, and responses of 1-3 were treated as "no" responses. Hits and false alarms were indexed in the same manner as in Experiment 1, with responses of 4-6 ("yes") being hits for pairs that had been presented in List 2 , but false alarms for pairs that had not been presented in List 2. Results from analyses that examined the full range of responses (1-6) did not provide useful additional information and so are not reported.

Preliminary analyses of the results from the differentiation and reminding groups revealed no significant interactions or main effects. That is, our attempt to gain task control over remindings was unsuccessful. In contrast, looking-back procedures have successfully been used to produce task control over recursive remindings due to the detection of shared category memberships of words (Jacoby \& Wahlheim, 2013), as well as those due to the detection of repetitions of words (Wahlheim, Maddox, \& Jacoby, under review). It seems likely that stronger procedures, such as the looking-back procedures, could also be devised to gain task control over recursive remindings produced by detection of change. The ability to gain task control over remindings will be important for a distinction between spontaneous and task-controlled remindings (for a discussion of this issue, see Jacoby \& Wahlheim, 2013), and doing so for recollection of change would provide further evidence that effects attributed to recursive reminding are not due to item differences.
Because of the ineffectiveness of the manipulation, data for the differentiation and remindings groups were collapsed for the following analyses. As will be shown, results of the analyses of hits and false alarms in Experiment 2 replicated the results found in Experiment 1, with the primary difference being that overall performance was lower in Experiment 2. The reduction in overall performance might have been due to any one or more of the changes in procedure across experiments: self-paced versus fixed rate of study, deletion of explicit detection of change during List 2, and insertion of the invisibility task between Lists 1 and 2 .

As in Experiment 1, the probability of a hit on repetition items was very high, whereas the probability of a false alarm to new foils was quite low (.86 vs. .07). Of primary interest were differences in list discrimination in the changed and control conditions. Figure 1 shows that the probability of a hit was higher in the changed-target condition than in the control-target condition (.80 vs. .72), $t(35)=3.62, p=.001$, whereas no difference in false alarms was apparent between the changed-foil and control-foil conditions (.40 vs. .43), $t(35)=-0.73, p=.47$. The list discrimination measure showed better discrimination for the changed than for the control conditions ( 1.22 vs. 0.84$), t(35)=2.79, p=.009$, indicating proactive facilitation for changed pairs.

The aggregate estimates of change (34\%) were less than the actual percentage of changed items $(50 \%), t(35)=-6.44$, $p<.001$, and aggregate estimates of repetition (30\%) were greater than the actual percentage of repeated items $(25 \%)$, $t(35)=2.38, p=.02$.

Change recollection and list discrimination Unlike in Experiment 1, the overall probabilities of participants reporting that responses had changed across lists at the time of test did not differ in the changed-foil and changed-target conditions (.59 vs. .61), $t(35)=-0.79, p=.44$. This lack of a difference suggests that requiring participants to detect change explicitly during List 2 in Experiment 1 made it likely that change would be detected for the first time at test and was responsible for the finding of a difference in change recollection between the changed conditions in Experiment 1 . The probability of mistakenly reporting that pairs from the control conditions had been changed was much higher than in Experiment 1, but did not differ between the control-foil and control-target conditions (.19 vs. .21), $t(35)=-0.95, p=.35$.

As in Experiment 1, we expected list discrimination in the changed conditions to reflect a mixture of proactive facilitation and proactive interference. Analyses of list discrimination conditionalized on recollection of change (Fig. 2) revealed that the probability of a hit for changedtarget pairs in list discrimination performance was higher when participants recollected change at test than when they did not (.83 vs. .76), and was also higher than the probability of a hit for control-target pairs (.83 vs. .72), $t \mathrm{~s}(35) \geq 2.51$, 
$p \mathrm{~s}<.02$. When change was not recollected, the probabilities of a hit did not differ between changed-target and controltarget pairs (.76 vs. .72), $t(35)=1.45, p=.16$. Examination of the probabilities of false alarms revealed many fewer false alarms in list discrimination performance to changedfoil test pairs when change was recollected than when it was $\operatorname{not}(.29$ vs. .61$), t(35)=-6.08, p<.001$. Fewer false alarms occurred for changed-foil pairs when change was recollected than for control-foil pairs (.29 vs. .43), $t(35)=-3.41, p=$ .002 , but the opposite was true when change was not recollected (.43 vs. .61), $t(35)=-4.05, p<.001$.

Most importantly, list discrimination replicated the results from Experiment 1 by showing proactive facilitation when change was recollected and proactive interference when change was not. As measured by $d^{\prime}$, list discrimination was higher in the changed conditions when change was recollected than in the control conditions (1.70 vs. 0.84$)$, $t(35)=5.81, p<.001$, but was lower than in the control conditions when change was not recollected ( 0.39 vs. 0.80$)$, $t(32)=-2.87, p=.007$.

Regression analyses The right of Table 3 displays the results from hierarchical multiple regression analyses in Experiment 2 that were conducted in the same manner as in Experiment 1. An analysis conducted at the item level revealed a pattern of results that was similar to those found in Experiment 1. Item differences did not account for a significant proportion of variance in list discrimination at the first step. However, after controlling for the contribution of item differences, change recollection significantly improved prediction at the second step. These results show that the effects of conditionalizing on change detection when examining list discrimination cannot be explained as being due to item selection effects. As we found in Experiment 1, differences among items in the extents to which they supported change recollection were a determinant of list discrimination performance in the changed conditions.

At the participant level, the results revealed that general discrimination ability explained a significant proportion of the variance in list discrimination performance for changed pairs. However, prediction was greatly improved by including a measure of change recollection at the second step, as in Experiment 1, again providing evidence for individual differences in the use of change recollection as a basis for list discrimination.

\section{General discussion}

The results from our experiments provide evidence that recollection of change can serve as a basis for list discrimination performance. Effects of the recollection of change on list discrimination converged with cued-recall results (Wahlheim \& Jacoby, 2013) by showing proactive facilitation when change was recollected at test and proactive interference when change was not recollected (for a similar pattern of results, see also Jacoby \& Wahlheim, 2013). The convergence of results was observed although our measure of recollection of change in the present experiments differed from that employed in earlier experiments: Rather than asking participants about change at test, as was done in the present experiments, earlier experiments had used a remindings-report procedure to measure recollection of change. The convergence of results across procedures for measuring the recollection of change provides support for the validity of the remindings-report procedure and also provides evidence that recollection of change can be measured by the more direct means of questioning memory for change at the time of test.

Although performance was poorer in Experiment 2 than in Experiment 1, the convergence of results across the experiments shows that neither requiring participants to explicitly detect change during List 2 nor the differences in study time in Experiment 1 was necessary to elicit effects of the recollection of change on list discrimination performance. Regression analyses at the level of items revealed that item differences contributed little to list discrimination performance, with the contribution being significant only in Experiment 1. However, differences among items in the extents to which they supported recollection of change did contribute to list discrimination performance. Similar analyses at the level of participants revealed large individual differences in the contributions of recollection of change to list differentiation performance.

At test, recollection of change enhanced list discrimination both by increasing the probability of correctly accepting pairs that had occurred in List 2 as having done so (hits), and by decreasing false alarms to items that had occurred in List 1 . When change was recollected, false alarms were lower than in the control condition because recollection of change provides a sure basis for rejecting List 1 pairs. When change was not recollected, the probability of a false alarm was much higher than in the control condition. The latter finding can be explained as resulting from the left-hand member of changed pairs being more familiar than those in the control condition, due to their repetition across lists. When unopposed by recollection of change, this greater familiarity was attributed to presentation in List 2, and so produced a high level of false alarms.

It is likely that differences in familiarity contributed to list discrimination performance in both experiments. List 2 items would be expected to be more familiar than List 1 items because of their more recent presentation, allowing familiarity to serve as a valid basis for hits (Yonelinas, 2001). However, differences in familiarity do not provide a sure basis 
for avoiding false alarms, whereas recollection of change does so. To account for the results that we have attributed to memory for change, it might be argued that retrieval of a List 1 pair during the presentation of a List 2 pair with which it shared a left-hand member served to strengthen the association of the List 1 pair to its List 1 context, and thereby reduced false alarms. However, one could as well argue that retrieval of List 1 pairs during the presentation of List 2 pairs would result in their becoming more familiar and associated with the List 2 context, with the result that the accuracy of list discrimination would be reduced. The full pattern of results is easily explained by appealing to effects of recollection of change, but difficult to interpret in terms of differences in either familiarity or the strength of simple associations between pairs and their list context.

It seems untenable to argue that people cannot remember relationships but, instead, can only form simple associations (e.g., Asch, 1969). Criss and Shiffrin (2005) have provided a useful review of research showing the importance of memory for higher-order relationships as compared to simple associations, and provided evidence of reliance on higher-order relationships for list discrimination. The results of the present experiments join those from earlier experiments in showing that a higher-order relationship (recollection of change) can serve as a basis for list discrimination.

The probability of detecting that the responses paired with an item were changed across lists was surprisingly low (.57), even in Experiment 1, which strongly encouraged detection of change. Differences among items, along with individual differences and task demands, are likely important for the detection of change. The recollection of change depends on the initial detection of change and individual differences, and is likely to also depend on the cues provided at the time of test. How detailed is the representation of an earlier event in the recursive reminding that is produced by detection of change? The results of the present experiments suggest that the right-hand member of the List 1 pair was included in the recursive representation of change. Otherwise, recollection of change could not serve as a basis for rejecting List 1 responses for the test of list discrimination. However, a recursive reminding created by change might sometimes only include that something was changed, rather than the particular change that occurred. As a commonplace example, when encountering an acquaintance who typically wears glasses but is not doing so on the particular occasion, we might detect that something has changed without being able to specify what has changed, with this being reflected by the recursive memory representation created by the detection of change. The results from the present experiments might be taken as evidence that recursive memory included a representation of the list membership of the List 1 pair involved in a reminding. However, this need not be the case. Instead, the recursive trace might only preserve information about the temporal order of events and not include the list in which the earlierpresented item had occurred. It seems likely that the completeness of the description of an earlier event that is included in the memory representation of recursive reminding varies across situations. More research will be needed to address this possibility.

Recollection of change is important for applied purposes as well as for purposes of theory. As an example, Loftus (1979) demonstrated the importance of conditions that lead to the detection of change for eliminating misinformation effects (i.e., retroactive interference). In her experiments, participants were presented with a slide show of an event, and then read a narrative about the event that included a few pieces of information that had been changed. When contradictions between the slide show and narrative were subtle, participants showed a misinformation effect by recalling information in the narrative as having been gained from the slide show. However, when a blatant contradiction was present in the narrative, participants were able to notice the contradiction and avoid misinformation effects. Zhu et al. (2010) showed that individual differences in the susceptibility to misinformation effects correlated with differences in change detection in a perceptual task. As a second example, encouraging the detection and recollection of contradiction (change) has been shown to be important. Otero and Kintsch (1992) embedded contradictory sentences in texts. The results of their experiment showed that many participants failed to detect the contradictions, but that one cluster almost always did so. Those who failed to detect contradictions later recalled either only one or neither of the contradictory statements. In contrast, those who detected contradictions showed much higher recall of both contradictory statements, producing results that are in accord with those found in our experiments. These are just a few examples that illustrate the potential importance of the detection and recollection of change for understanding phenomena across a broad range of domains that have otherwise been treated as largely separate.

Investigation of effects of the detection and recollection of change hold promise in a variety of domains in which they have not yet been investigated. For example, detection and recollection of change might be important for correction of errors (e.g., Butterfield \& Metcalfe, 2001). As a second example, older adults are more prone to proactive interference than are young adults, and that difference has been shown to result from a lessened ability to recollect, in combination with preserved automatic influences of memory (e.g., Jacoby, Bishara, Hessels, \& Toth, 2005). Perhaps the form of recollection that is important is recollection of change. More generally, to adapt successfully to a continually changing world, an important first step might be the detection and recollection of change. 
Author note This research was supported by a James S. McDonnell Foundation 21st Century Science Initiative in Bridging Brain, Mind, and Behavior Collaborative Award to the first author. We thank Emily Gardner and Rachel Teune for their assistance with data collection.

\section{References}

Anderson, J. R., \& Bower, G. H. (1974). Interference in memory for multiple contexts. Memory \& Cognition, 2, 509-514.

Asch, S. E. (1969). A reformulation of the problem of associations. American Psychologist, 24, 92-102.

Butterfield, B., \& Metcalfe, J. (2001). The correction of errors committed with high confidence. Metacognition and Learning, 1, $1556-1623$.

Criss, A. H., \& Shiffrin, R. M. (2005). List discrimination in associative recognition and implications for representation. Journal of Experimental Psychology: Learning, Memory, and Cognition, 31, 1199-1212. doi:10.1037/0278-7393.31.6.1199

Hintzman, D. L. (2010). How does repetition affect memory? Evidence from judgments of recency. Memory \& Cognition, 38, 102-115. doi:10.3758/MC.38.1.102

Jacoby, L. L. (1996). Dissociating automatic and consciously controlled effects of study/test compatibility. Journal of Memory and Language, 35, 32-52.

Jacoby, L. L., Bishara, A. J., Hessels, S., \& Toth, J. P. (2005). Aging, subjective experience, and cognitive control: Dramatic false remembering by older adults. Journal of Experimental Psychology. General, 134, 131-148. doi:10.1037/0096-3445.134.2.131

Jacoby, L. L., \& Wahlheim, C. N. (2013). On the importance of looking back: The role of recursive remindings in recency judgments and cued recall. Memory \& Cognition. doi:10.3758/ s13421-013-0298-5

Loftus, E. F. (1979). Reactions to blatantly contradictory information. Memory \& Cognition, 7, 368-374.
Macmillan, N. A., \& Creelman, C. D. (2005). Detection theory: A user's guide (2nd ed.). Mahwah: Erlbaum.

Murnane, K., Phelps, M. P., \& Malmberg, K. (1999). Contextdependent recognition memory: The ICE theory. Journal of Experimental Psychology. General, 128, 403-415. doi:10.1037/ 0096-3445.128.4.403

Nelson, D. L., McEvoy, C. L., \& Schreiber, T. A. (1998). The University of South Florida word association, rhyme, and word fragment norms [Database]. Retrieved from http://w3.usf.edu/ FreeAssociation/

Otero, J., \& Kintsch, W. (1992). Failures to detect contradictions in a text: What readers believe versus what they read. Psychological Science, 3, 229-235.

Postman, L., \& Underwood, B. J. (1973). Critical issues in interference theory. Memory \& Cognition, 1, 19-40.

Sahakyan, L., \& Kelley, C. M. (2002). A contextual change account of the directed forgetting effect. Journal of Experimental Psychology: Learning, Memory, and Cognition, 28, 1064-1072. doi:10.1037/0278-7393.28.6.1064

Tzeng, O. J. L., \& Cotton, B. (1980). A study-phase retrieval model of temporal coding. Journal of Experimental Psychology: Human Learning and Memory, 6, 705-716.

Wahlheim, C. N., \& Jacoby, L. L. (2013). Remembering change: The critical role of recursive remindings in proactive effects of memory. Memory \& Cognition, 41, 1-15. doi:10.3758/s13421-012-0246-9

Winograd, E. (1968). List differentiation as a function of frequency and retention interval. Journal of Experimental Psychology, 76 (2, Pt. 2), 1-18. doi:10.1037/h0025380

Winograd, E., \& Soloway, R. M. (1985). Reminding as a basis for temporal judgments. Journal of Experimental Psychology: Learning, Memory, and Cognition, 11, 262-271.

Yonelinas, A. P. (2001). Consciousness, control, and confidence: The 3 Cs of recognition memory. Journal of Experimental Psychology. General, 130, 361-379. doi:10.1037/0096-3445.130.3.361

Zhu, B., Chen, C. C., Loftus, E. F., Lin, C., He, Q., Chen, C., et al. (2010). Individual differences in false memory from misinformation: Cognitive factors. Memory, 18, 543-555. doi:10.1080/09658211. 2010.487051 